\title{
Latest Results on Radiation Tolerance of Diamond Detectors
}

\section{The RD42 Collaboration}

A. Oh ${ }^{24 *}$ A. Alexopoulos, ${ }^{3}$, M. Artuso ${ }^{20}$, F. Bachmair ${ }^{24}$, L. Bäni ${ }^{24}$, M. Bartosik ${ }^{3}$, J. Beacham ${ }^{13}$, H. Beck ${ }^{23}$, V. Bellini' ${ }^{2}$, V. Belyaev ${ }^{12}$, B. Bentele ${ }^{19}$, A. Bes ${ }^{27}$, J-M. Brom ${ }^{7}$, M. Bruzzi ${ }^{4}$, G. Chiodini' ${ }^{26}$, D. Chren ${ }^{18}$, V. Cindro ${ }^{11}$, G. Claus ${ }^{7}$, J. Collot ${ }^{27}$, J. Cumalat ${ }^{19}$, A. Dabrowski ${ }^{3}$, R. D'Alessandro ${ }^{4}$, D. Dauvergne ${ }^{27}$, W. de Boer ${ }^{10}$, S. Dick ${ }^{13}$, C. Dorfer ${ }^{24}$, M. Dünser ${ }^{3}$, V. Eremin ${ }^{6}$, G. Forcolin ${ }^{22}$, J. Forneris ${ }^{15}$, L. Gallin-Martel ${ }^{27}$, M-L. Gallin-Martel ${ }^{27}$, K.K. Gan ${ }^{13}$, M. Gastal ${ }^{3}$, M. Goffe ${ }^{7}$, J. Goldstein ${ }^{17}$, A. Golubev ${ }^{8}$, A. Gorišek ${ }^{9}$, E. Grigoriev ${ }^{8}$, J. Grosse-Knetter ${ }^{23}$, A. Grummer ${ }^{21}$, M. Guthoff ${ }^{3}$, I. Haughton ${ }^{22}$, B. Hiti ${ }^{9}$, D. Hits ${ }^{24}$, M. Hoeferkamp ${ }^{21}$, T. Hofmann ${ }^{3}$, J. Hosslet ${ }^{7}$, J-Y. Hostachy ${ }^{27}$, F. Hügging ${ }^{1}$, C. Hutton ${ }^{17}$, J. Janssen ${ }^{1}$, H. Kagan ${ }^{13}$, K. Kanxheri' ${ }^{28}$, G. Kasieczka ${ }^{24}$, R. Kass ${ }^{13}$, F. Kassel ${ }^{10}$, M. Kis ${ }^{5}$, G. Kramberger ${ }^{9}$, S. Kuleshov ${ }^{8}$, A. Lacoste ${ }^{27}$, S. Lagomarsino ${ }^{4}$, A. Lo Giudice ${ }^{15}$, E. Lukosi ${ }^{25}$, C. Maazouzi ${ }^{7}$, I. Mandic ${ }^{9}$, C. Mathieu ${ }^{7}$, M. Menichelli ${ }^{28}$, M. Mikuž ${ }^{9}$, A. Morozzi ${ }^{28}$, J. Moss $^{29}$, R. Mountain ${ }^{20}$, S. Murphy ${ }^{22}$, P. Olivero ${ }^{15}$, D. Passeri ${ }^{28}$, H. Pernegger ${ }^{3}$, R. Perrino ${ }^{26}$, F. Picollo ${ }^{15}$, M. Pomorski ${ }^{11}$, R. Potenza ${ }^{2}$, A. Quadt ${ }^{23}$, A. $\mathbf{R e}^{15}$, M. Reichmann ${ }^{24}$, G. Riley ${ }^{25}$, S. Roe $^{3}$, D. Sanz ${ }^{24}$, M. Scaringella ${ }^{4}$, C.J. Schmidt ${ }^{5}$, S. Schnetzer ${ }^{14}$, S. Sciortino ${ }^{4}$, A. Scorzoni ${ }^{28}$, S. Seidel ${ }^{21}$, L. Servoli ${ }^{28}$, D.S. Smith ${ }^{13}$, B. Sopko ${ }^{18}$, V. Sopko ${ }^{18}$, S. Spagnolo ${ }^{26}$, S. Spanier ${ }^{25}$, K. Stenson ${ }^{19}$, R. Stone ${ }^{14}$, C. Sutera ${ }^{2}$, A. Taylor ${ }^{21}$, M. Traeger ${ }^{5}$, D. Tromson ${ }^{11}$, W. Trischuk ${ }^{16}$, C. Tuve $^{2}$, J. Velthuis ${ }^{17}$, N. Venturi ${ }^{3}$, E. Vittone ${ }^{15}$, S. Wagner $^{19}$, R. Wallny ${ }^{24}$, J.C. Wang ${ }^{20}$, J. Weingarten ${ }^{23}$, C. Weiss ${ }^{3}$, N. Wermes ${ }^{1}$, M. Yamouni ${ }^{27}$, M. Zavrtanik ${ }^{9}$

${ }^{1}$ Universität Bonn, Bonn, Germany; ${ }^{2}$ INFN/University of Catania, Catania, Italy; ${ }^{3}$ CERN, Geneva, Switzerland; ${ }^{4}$ INFN/University of Florence, Florence, Italy; ${ }^{5}$ GSI, Darmstadt, Germany; ${ }^{6}$ Ioffe Institute, St. Petersburg, Russia; ${ }^{7}$ IPHC, Strasbourg, France; ${ }^{8}$ ITEP, Moscow, Russia; ${ }^{9}$ Jožef Stefan Institute, Ljubljana, Slovenia; ${ }^{10}$ Universität Karlsruhe, Karlsruhe, Germany; ${ }^{11}$ CEA-LIST Technologies Avancées, Saclay, France; ${ }^{12}$ MEPHI Institute, Moscow, Russia; ${ }^{13}$ The Ohio State University, Columbus, OH, USA; ${ }^{14}$ Rutgers University, Piscataway, NJ, USA; ${ }^{15}$ University of Torino, Torino, Italy; ${ }^{16}$ University of Toronto, Toronto, ON, Canada; ${ }^{17}$ University of Bristol, Bristol, UK; ${ }^{18}$ Czech Technical Univ., Prague, Czech Republic; ${ }^{19}$ University of Colorado, Boulder, CO, USA; ${ }^{20}$ Syracuse University, Syracuse, NY, USA; ${ }^{21}$ University of New Mexico, Albuquerque, NM, USA; ${ }^{22}$ University of Manchester, Manchester, UK; ${ }^{23}$ Universität Göttingen, Göttingen, Germany; ${ }^{24}$ ETH Zürich, Zürich, Switzerland; ${ }^{25}$ University of Tennessee, Knoxville, TN, USA; ${ }^{26}$ INFN-Lecce, Lecce, Italy; ${ }^{27}$ LPSC-Grenoble, Grenoble, France; ${ }^{28}$ INFN-Perugia, Perugia, Italy; ${ }^{29}$ California State University, Sacramento, CA, USA.

The 39th International Conference on High Energy Physics (ICHEP2018)

4-11 July, 2018

Seoul, Korea

${ }^{*}$ Corresponding author. Email:alexander.oh@manchester.ac.uk 
At present most experiments at the CERN Large Hadron Collider (LHC) are planning upgrades in the next 5-10 years for their innermost tracking layers as well as luminosity monitors to be able to take data as the luminosity increases and CERN moves toward the High Luminosity-LHC (HL-LHC). These upgrades will most likely require more radiation tolerant technologies than exist today. As a result this is one area of intense research, and Chemical Vapour Deposition (CVD) diamond is one such technology. CVD diamond has been used extensively in beam condition monitors as the innermost detectors in the highest radiation areas of all LHC experiments. This talk describes the preliminary radiation tolerance measurements of the highest quality polycrystalline CVD material for a range of proton energies and neutrons obtained with this material with the goal of elucidating the issues that should be addressed for future diamond based detectors. The talk presents the evolution of various semiconductor parameters as a function of dose.

\section{Introduction}

The innermost tracking detector systems at Hadron Collider Experiments of the next generation will face a challenging radiation environment. Synthetic diamond from a chemical vapour deposition process (CVD) has been investigated as a promising alternative candidate to silicon due to its nearly twice higher atomic displacement energy and high intrinsic resistivity.

The RD42 collaboration has explored the radiation tolerance of single (sCVD) and polycrystalline CVD diamond (pCVD) with protons and neutrons at energy ranges relevant to the particle spectra encountered at the Large Hadron Collider (LHC), up to a particle fluence required by the high luminosity upgrade of the LHC (HL-LHC) [1]. In the following the characterisation method of radiation tolerance will be discussed (Section 2) and the results of protons and neutron irradiations on pCVD and sCVD will be summarised (Section 3).

\section{Characterisation of Radiation Tolerance}

Diamond samples were characterised before and after irradiation by measuring the charge signal with $120 \mathrm{GeV} / \mathrm{c}$ protons in the H6 beam line at the CERN Super-Proton-Synchrotron (SPS). The samples were typically sized $5 \mathrm{~mm} \times 5 \mathrm{~mm}$ or $10 \mathrm{~mm} \times 10 \mathrm{~mm}$ and metallized with a segmented strip electrode structure on the front-side and a continuous electrode on the back-side. The strips were wire-bonded to a VA2 readout chip for charge integration and shaping the signal; the electronic noise was about 80 electrons. Before testing the sensors they were expose to beta particles from a ${ }^{90} \mathrm{Sr}$ source to fill charge traps. The detectors were tested at an electric field strength of $1 \mathrm{~V} \mu \mathrm{m}^{-1}$ and $2 \mathrm{~V} \mu \mathrm{m}^{-1}$. A beam telescope predicted the track position at the diamond sensor with a resolution of $4 \mu \mathrm{m}$. The charge signal was built from the sum of the highest two strip cluster within 10 strips around the predicted track position.

A $120 \mathrm{GeV} / \mathrm{c}$ proton is a minimum ionising particle (MIP) and will create on average 36 electron-hole pairs per micrometer in diamond. The measured MIP signal $Q_{m}$ is commonly expressed in terms of the charge collection distance defined as $\delta=\frac{Q_{m}}{36 e \mu \mathrm{m}^{-1}}$. The charge signal is reduced by active traps reducing the life-time of the electrons and holes. The life time $\tau$ is proportional to the "Schubweg" $\lambda \propto \frac{1}{\tau}$ and since production of traps by radiation is proportional to the 
fluence the radiation damage can be parameterised as

$$
\frac{1}{\lambda}=\frac{1}{\lambda_{0}}+k_{\lambda} \Phi
$$

with $\lambda_{0}$ representing the un-irradiated state, and the damage constant $k_{\lambda}$ describing the introduction of additional traps with the fluence $\Phi$. The MIP signal efficiency $\varepsilon$, charge collection distance $\delta$ and Schubweg are related by

$$
\varepsilon=Q_{m} / Q_{M I P}=\delta / d=2 \frac{\lambda}{d}\left(1-\frac{\lambda}{d}\left(1-e^{-d / \lambda}\right)\right)
$$

with $d$ the thickness of the detector.

A typical signal spectrum obtained with $120 \mathrm{GeV} / \mathrm{c}$ protons with single crystal CVD diamond material is shown in Figure 1a. The spectra show the typical Landau shape for MIP signals, the degradation of the signal with proton fluence is clearly visible. In Figure $1 \mathrm{~b}$ the dependence of the ratio, $f$, of FWHM to most probable value is compared for single crystal and polycrystalline CVD diamond. The value of $f$ is decreasing for pCVD while sCVD shows a slow rise of $f$ with proton fluence, indicating a common value at high proton fluence. The resistivity of diamond is not significantly changed with irradiation fluence [2], and due to the large band shows also no significant dependence with temperature at room temperatures or below [2].

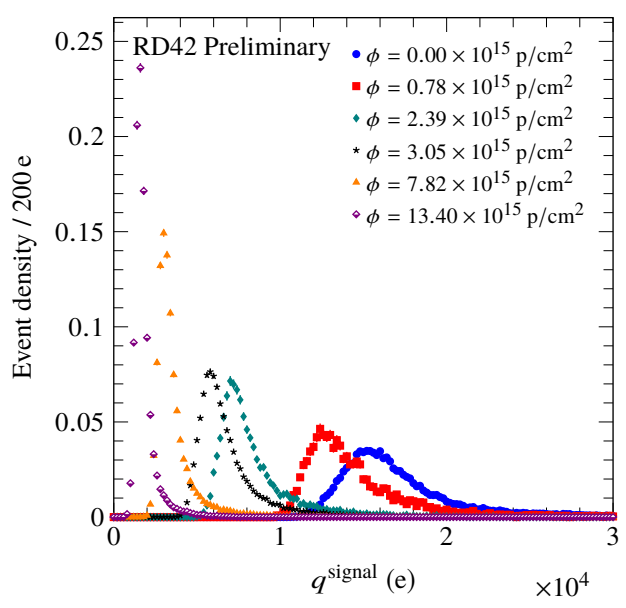

(a)

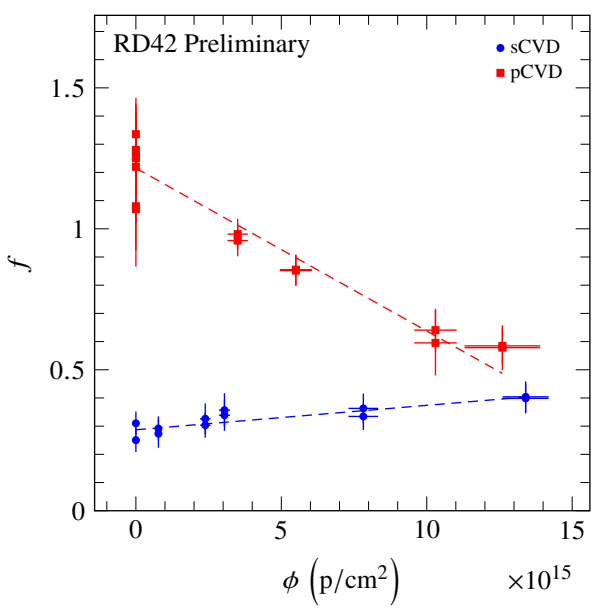

(b)

Figure 1: MIP signal spectra of single crystal diamond detectors at different $800 \mathrm{MeV}$ proton fluences (a), and ratio of FWHM to the most probable value of the MIP signal for single crystal and polycrystalline CVD diamond (b) [3].

From the measured charge $Q_{m}$ the Schubweg $\lambda$ can be determined by solving equation 2.2. The linear dependence of the inverse of lambda, $1 / \lambda$, with proton fluence can be seen in Figure 2a. The slope of a linear fit to these data in $1 / \lambda$ allows to extract the damage constant $k_{\lambda}$ from equation 2.1. The gradient of $1 / \lambda$ is comparable for different SCVD and pCVD diamond samples while the variations in offset are due to the initial material conditions and are captured by the $\lambda_{0}$ term in equation 2.1. This indicates that the simple model for radiation damage parametrises well the damage in PCVD and SCVD diamond. 


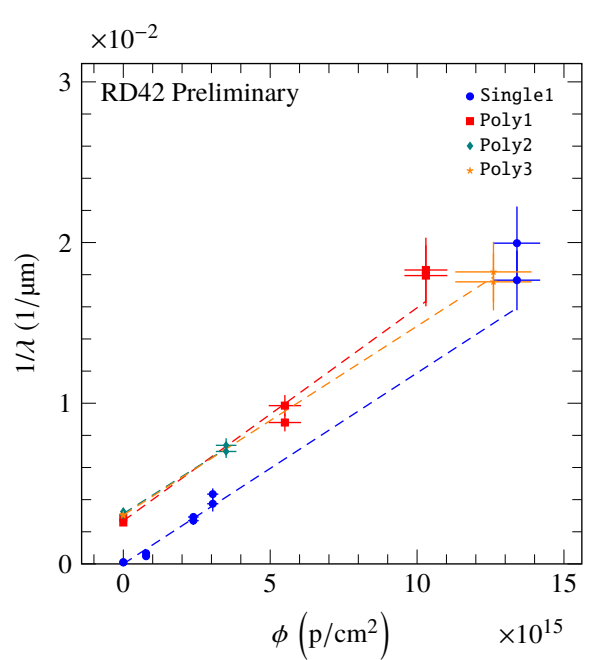

(a)

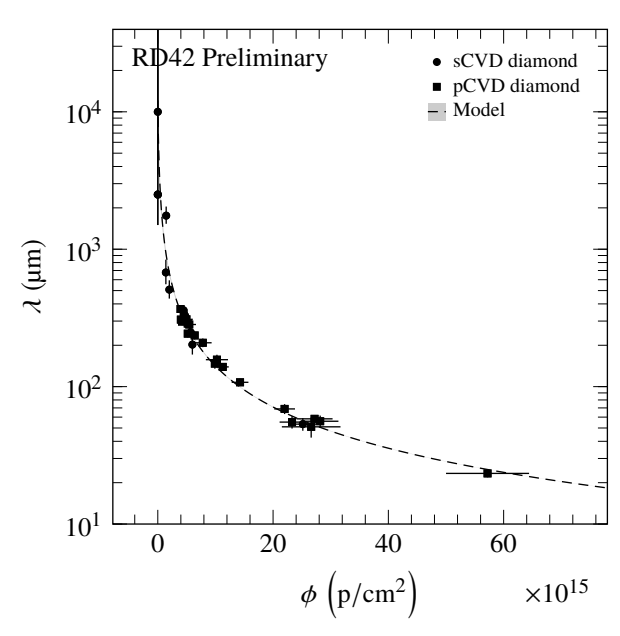

(b)

Figure 2: Measurements of inverse Schubweg as a function of $800 \mathrm{MeV}$ protons fluence for SCVD and pCVD samples for two polarities with statistical and systematic error added in quadrature [3] (a). Measured Schubweg as a function of $24 \mathrm{GeV}$ protons equivalent fluence compared to the radiation damage model (dashed curve) [3] (b).

\section{Summary of Results}

A series of irradiations of sCVD and pCVD diamonds and subsequent characterisation in testbeams has been conducted. Samples have been irradiated with $25 \mathrm{MeV}$ to $24 \mathrm{GeV}$ protons, up to maximum fluence of $1.3 \cdot 10^{16} \mathrm{p} \mathrm{cm}^{-2}$ and with fast neutrons corresponding to an energy range of $1 \mathrm{MeV}$ to $10 \mathrm{MeV}$ up to a fluence of $1.3 \cdot 10^{16} \mathrm{n} \mathrm{cm}^{-2}$.

From this data-set the damage constants $k$ as a function of particle type and energy were extracted. Although we are not in a position at this time to quote the absolute values of $k$, current preliminary values indicate they are 2-4 times lower in diamond than in silicon. The initial signal is lower in diamond compared to silicon, however the low leakage current and low $k$ factors puts diamond ahead of silicon for fluence above $10^{15}$ particles $\mathrm{cm}^{2}$ in terms of signal-to-noise ratio for a typical HL-LHC pixel detector application [4].

\section{References}

[1] G. Apollinari, O. Brüning, T. Nakamoto and L. Rossi, High Luminosity Large Hadron Collider HL-LHC, CERN Yellow Report (2015) 1 [1705. 08830].

[2] RD42 collaboration, S. Seidel, Recent results on diamond radiation tolerance, JINST 9 (2014) C01013.

[3] L. Baeni, Top Quarks and Diamonds, Ph.D. thesis, ETH, Zuerich, 2017. http://doi.org/10.3929/ethz-a-010748643.

[4] J.-W. Tsung, M. Havranek, F. Hügging, H. Kagan, H. Krüger and N. Wermes, Signal and noise of diamond pixel detectors at high radiation fluences, JINST 7 (2012) P09009. 